Research Article

\title{
Mortality in acute stroke: predictive value of lipid profile and routine investigations
}

\author{
Prakash Chandra Pandey*, Naresh Chandra Dwivedi, Rakesh Kumar Yadav, \\ Kamlesh Kumar Sonkar
}

Department of Medicine, MLN Medical College, Allahabad, U.P, India

Received: 21 June 2015

Revised: 22 June 2015

Accepted: 11 July 2015

*Correspondence:

Dr. Prakash Chandra Pandey,

E-mail: drprakashpandey@gmail.com

Copyright: ( $)$ the author(s), publisher and licensee Medip Academy. This is an open-access article distributed under the terms of the Creative Commons Attribution Non-Commercial License, which permits unrestricted non-commercial use, distribution, and reproduction in any medium, provided the original work is properly cited.

\begin{abstract}
Background: The prognostic value of routine blood investigations in acute stroke has not been adequately studied. Most studies do not use accumulative measurement of these parameters and numbers of subjects taken were less. In this study we assess the impact of these simple parameters on the mortality in acute stroke patients as well as their correlation with clinical findings.

Methods: Total 205 consecutive patients of acute stroke presented within 72 hours of symptom onset were studied. After clinical evaluation and neuroimaging, routine blood investigations along with lipid profile estimations were performed. The patients were followed up for a maximum period of 30 days from onset of stroke.

Results: After analysis it was demonstrated that out of all clinical parameters low Glasgow Coma Scale (GCS), higher systolic and diastolic blood pressure can strongly predict fatality significantly. Among blood investigations raised leukocyte counts(TLC), erythrocyte sedimentation rate, blood urea, serum creatinine, serum bilirubin along with decreased level of total cholesterol $(\mathrm{p}=0.0001)$ and low density lipoprotein (LDL) ( $\mathrm{p}=0.02)$ signifies their accumulative importance in predicting fatality in expired patients. But after logistic regression analysis only GCS, TLC, blood urea, serum creatinine and total cholesterol independently correlate with mortality.

Conclusions: Our study shows that it is useful to take necessary interventions, accordingly in early hours focusing on correctable parameters in acute stroke to prevent mortality in acute stroke.
\end{abstract}

Keywords: Acute stroke, Lipid profile, Mortality

\section{INTRODUCTION}

Acute stroke is a medical emergency which can cause permanent neurological damage, complications and even may leads to death. Results of stroke vary widely depending on the size and location of the lesions. It is a heterogeneous condition with respect to prognosis. It is not possible to predict exact outcome of stroke with accuracy. The chances of survival in stroke depend on a great variety of variables. Globally, approximately 15 million new acute stroke events occur every year and approximately 55 million people had stroke at some time in the past. Two thirds of these individuals live in lowand middle-income countries such as India. By 2050, it is anticipated that $80 \%$ of stroke events will occur in people living in these regions. ${ }^{1}$

A recent Indian study have shown different prevalence rates in rural and urban areas (rural areas 84262/100,000; urban areas 334-424/100,000) with incidence rate of $119-145 / 100,000$ and 30 day case fatality was found to be $41.08 \% .^{2}$ According to Indian collaborative acute stroke study (2002-2004) with 2162 cases, stroke incidence higher in age group of 41-70 
years having major risk factor hypertension alone or also along with Diabetes mellitus (DM) and Coronary Artery Disease(CAD). Maximum number of patients $(77 \%)$ of this study was of acute ischemic stroke. ${ }^{3}$ Risk factors for acute stroke may be non-modifiable -Age, gender (male $>$ female), race (Afro-Caribbean > Asian > European), heredity and previous vascular events (CAD, stroke or peripheral embolism). The modifiable factors are high blood pressure, heart disease (atrial fibrillation, heart failure, and endocarditis), DM, Dyslipidemia, smoking, excess alcohol consumption, polycythemia, oral contraceptives and social deprivation.

Predicting outcome after stroke is a question which is still unanswered. Experimental and clinical research shows the importance of early hours in acute stroke. It is the most important time for effective intervention. Most of studies focusing on conventional risk factors, clinical condition at presentation and on radiological parameters. Various studies have been undertaken to assess the prognosis of stroke evaluating different clinical and biochemical parameters. Most of the scoring systems used to determine outcome in stroke consider only the neurological deficit related parameters when evaluating these patients. While in our study we take into consideration various hematological, biochemical parameters and lipid profile in addition to neurological deficit, thus it may be a better alternative and effective predictor of 30 day fatality in acute stroke.

\section{METHODS}

All patients who were consecutively admitted in an intensive care unit presenting within 72 hours of stroke onset, included in the study irrespective of age, sex or type of stroke, during July 2013 to December 2014. Acute stroke diagnosed by clinical findings and confirmed by non- contrast head computer tomography. Presentation more than 72 hours after the onset of the signs and symptoms of stroke, prior stroke, non-stroke causes of focal neurological deficit and patients those could not be followed up were excluded from study. Demographic information included gender and age; medical history regarding hypertension, diabetes mellitus (DM), hyperlipidemia, atrial fibrillation, smoking; prior stroke and coronary artery disease (CAD) were obtained. Information of medications at the time of stroke was collected, which includes use of statins, angiotensinconverting enzyme inhibitors, aspirin, clopidogril and warfarin.

Clinical evaluation regarding Glasgow coma scale(GCS), systolic blood pressure (SBP) and diastolic blood pressure (DBP); blood investigations including hemoglobin $(\mathrm{Hb})$, total leukocyte count (TLC), platelet count(PC), erythrocyte sedimentation rate (ESR), random blood sugar (RBS), blood urea, serum creatinine (S. creatinine), serum Sodium (S. Na), serum Potassium (S. $\mathrm{K}$ ), serum bilirubin, liver enzymes (aspartate transaminase - AST, alanine transaminase-ALT, alkaline
phosphatase-ALP), serum albumin (S. albumin), serum globulin (S. globulin) and lipid profile estimations performed along with non-contrast head computer tomography.

Being a prospective study the patients were followed up for a maximum period of 30 days from the onset of stroke. The outcome of the patients at the end of 30 days was determined as discharged and expired. Logistic regression analysis was carried out among the significant parameters to identify independent predictors of 30 days fatality. The statistical analysis was conducted by using the Statistical Package for the Social Sciences, version 11.0 (SPSS Inc., Chicago, IL, USA).

\section{RESULTS}

Among the total 205 cases in the study, 140 (68.29\%) patients discharged and $65(31.70 \%)$ patients expired during the hospital stay or during follow up. There were 130 males and 75 females, who satisfying inclusion criteria with mean age of $63.17 \pm 13.61$ years. In Table 1 distribution of patients according to age in both discharged and expired patients shown. Out of the total expired patients $61.53 \%$ were belonging to age group of $\geq 60$ years which show significance of age in prognosis $(\mathrm{p}=0.01)$.

In analysis of clinical parameters patients who expired had lower (6.07 \pm 3.32$)$ GCS and significantly higher SBP as well as DBP in comparison to discharged group. The p- values for GCS 0.0001, for SBP 0.049 and for DBP 0.0232 , all these values were statistically significant in between expired and discharged groups.

With regard to haematological parameters higher value of TLC and ESR observed in expired subgroup ( $\mathrm{p}$ - value $<0.05$ ). Mean value of $\mathrm{Hb}$ in discharged group was lower than expired one. PC high in discharged patients in comparison to expired patients, but these values were not significant in term of statistics. Correlation of biochemical parameters with respect to outcome is shown in table 2. In expired subgroup RBS, blood urea, S. creatinine, S. Na, S.K, S. bilirubin values were higher and in discharged subgroup AST, ALT, albumin, globulin were higher. But after applying unpaired t-test only blood urea, $\mathrm{S}$. creatinine and $\mathrm{S}$. bilirubin had significance with fatality (p - value <0.05) (Table 2).

Lipid profile estimation done of all patients of acute stroke and after analysing total cholesterol, low density lipoprotein ( LDL) were found to be higher in discharged group in comparison with expired group and both of these were statistically significant, $\mathrm{p}$ value 0.0001 and 0.02 respectively. Serum triglyceride (S.Tg), high density lipoprotein (HDL) and very low density lipoprotein (VLDL) did not show any statically significance (Table3). 
Multivariate logistic regression analysis was performed among the significant parameters and after analysis it was found that out of all observed parameters only GCS,
TLC, blood urea, S. creatinine and total cholesterol can predict outcome independently (Table 4).

Table 1: Distribution of cases according to outcome and age.

\begin{tabular}{|c|c|c|c|c|}
\hline Age (years) & Expired & Discharged & $X^{2}$ & p value \\
\hline$\leq 30$ & $5(7.6 \%)$ & - & \multirow{6}{*}{13.1276} & \multirow{6}{*}{0.010} \\
\hline $30-39$ & $5(7.6 \%)$ & $5(3.57 \%)$ & & \\
\hline $40-49$ & $5(7.6 \%)$ & $10(7.14 \%)$ & & \\
\hline $50-59$ & $10(15.38 \%)$ & $25(17.85 \%)$ & & \\
\hline$\geq 60$ & $40(61.53 \%)$ & $100(71.42 \%)$ & & \\
\hline Total & 65 & 140 & & \\
\hline
\end{tabular}

Table 2: Statistical analysis of clinical, hematological and biochemical parameters in acute stroke patients.

\begin{tabular}{|c|c|c|c|c|}
\hline Parameters & $\begin{array}{l}\text { Discharged }(\mathrm{n}=140) \\
(\text { Mean } \pm \text { SD })\end{array}$ & $\begin{array}{l}\text { Expired }(n=65) \\
(\text { Mean } \pm \text { SD })\end{array}$ & t-value & p-value \\
\hline GCS & $10.57 \pm 3.42$ & $6.07 \pm 3.32$ & 3.94 & 0.0001 \\
\hline SBP (mm Hg) & $148.30 \pm 33.41$ & $164.21 \pm 25.28$ & 1.69 & 0.049 \\
\hline $\mathrm{DBP}(\mathrm{mm} \mathrm{Hg})$ & $87.84 \pm 13.30$ & $95.64 \pm 10.28$ & 2.05 & 0.0232 \\
\hline $\mathrm{Hb}(\mathrm{g} / \mathrm{dL})$ & $11.47 \pm 2.23$ & $11.69 \pm 3.27$ & 0.45 & 0.65 \\
\hline $\operatorname{TLC}\left(/ \mathrm{mm}^{3}\right)$ & $9267.86 \pm 3429.62$ & $15646.15 \pm 2944.38$ & 5.78 & 0.0001 \\
\hline ESR (mm in $\left.1^{\text {st }} \mathrm{hr}.\right)$ & $23.18 \pm 8.94$ & $30.33 \pm 5.69$ & 2.54 & 0.01 \\
\hline PC $\left(\mathrm{lakh} / \mathrm{mm}^{3}\right)$ & $2.02 \pm 0.69$ & $1.68 \pm 0.72$ & 1.39 & 0.17 \\
\hline RBS (mg/dL) & $118.79 \pm 47.64$ & $134.00 \pm 61.21$ & 0.86 & 0.39 \\
\hline Blood urea $(\mathrm{mg} / \mathrm{dL})$ & $31.27 \pm 14.52$ & $46.10 \pm 21.43$ & 2.18 & 0.03 \\
\hline $\begin{array}{l}\text { S.creatinine } \\
(\mathrm{mg} / \mathrm{dL})\end{array}$ & $1.10 \pm 0.45$ & $2.07 \pm 0.60$ & 5.57 & 0.0001 \\
\hline S.Na (meq/L) & $139.29 \pm 7.10$ & $140.00 \pm 6.36$ & 0.30 & 0.75 \\
\hline S.K (meq/L) & $4.20 \pm 0.78$ & $4.46 \pm 0.76$ & 1.01 & 0.31 \\
\hline S.bilirubin (mg/dL) & $0.58 \pm 0.16$ & $1.16 \pm 0.94$ & 3.16 & 0.003 \\
\hline AST (IU/L) & $46.27 \pm 47.88$ & $35.63 \pm 11.74$ & 0.78 & 0.43 \\
\hline ALT (IU/L) & $45.80 \pm 41.08$ & $31.35 \pm 15.68$ & 1.22 & 0.22 \\
\hline S.albumin $(\mathrm{g} / \mathrm{dL})$ & $3.67 \pm 0.86$ & $3.62 \pm 0.55$ & 0.20 & 0.84 \\
\hline S.globulin $(\mathrm{g} / \mathrm{dL})$ & $3.50 \pm 1.03$ & $3.17 \pm 0.82$ & 0.98 & 0.33 \\
\hline
\end{tabular}

AST- Aspartate Transaminase; ALT- Alanine Transaminase; ALP- Alkaline Phosphatase; DBP- Diastolic Blood Pressure; ESR- Erythrocyte Sedimentation Rate; GCS- Glasgow Coma Scale; Hb- haemoglobin; PC- Platelet Count; RBS- Random Blood Sugar; SBP-Systolic Blood Pressure; S- Serum; K- Potassium; Na- Sodium; TLC- Total Leukocyte Count.

Table 3: Lipid profile in stroke subtypes according to outcome.

\begin{tabular}{|lllll|}
$\begin{array}{l}\text { Lipid } \\
\text { Profile }\end{array}$ & $\begin{array}{l}\text { Discharged }(\mathbf{n}=140) \\
(\text { Mean } \pm \text { SD })\end{array}$ & $\begin{array}{l}\text { Expired }(\mathbf{n}=65) \\
(\text { Mean } \pm \text { SD })\end{array}$ & $\begin{array}{l}\mathbf{t} \\
\text { value }\end{array}$ & $\begin{array}{l}\mathbf{p} \\
\text { value }\end{array}$ \\
\hline S. Triglyceride $(\mathrm{mg} / \mathrm{dL})$ & $130.60 \pm 107.42$ & $189.15 \pm 206.97$ & 1.19 & 0.23 \\
\hline Total Cholesterol $(\mathrm{mg} / \mathrm{dL})$ & $234.15 \pm 55.15$ & $159.80 \pm 28.69$ & 4.56 & 0.0001 \\
\hline HDL $(\mathrm{mg} / \mathrm{dL})$ & $47.56 \pm 8.77$ & $52.31 \pm 16.10$ & 1.22 & 0.22 \\
\hline LDL $(\mathrm{mg} / \mathrm{dL})$ & $114.39 \pm 61.91$ & $71.50 \pm 27.49$ & 2.29 & 0.0200 \\
\hline VLDL $(\mathrm{mg} / \mathrm{dL})$ & $26.09 \pm 21.49$ & $38.52 \pm 40.85$ & 1.28 & 0.20 \\
\hline
\end{tabular}

HDL- High Density Lipoprotein; LDL- Low Density Lipoprotein; S.Tg- Serum Triglyceride; VLDL- Very Low Density Lipoprotein. 
Table 4: Data obtained after applying multivariate logistic regression on significant parameters.

\begin{tabular}{|lllllll|} 
Parameter & B & S.E. & Sig. & OR & $\chi^{2}$ & $\begin{array}{l}\text { p } \\
\text { value }\end{array}$ \\
\hline GCS & 0.4567 & 0.1713 & 0.0077 & 1.5788 & 23.0118 & 0.0001 \\
\hline TLC & 0.0003 & 0.0165 & 0.0001 & 0.9997 & 12.4988 & 0.0019 \\
\hline Blood urea & 0.0954 & 0.0478 & 0.0460 & 1.1002 & 28.4193 & 0.00 \\
\hline S. creatinine & 4.2423 & 1.4810 & 0.0042 & 0.0144 & 28.4193 & 0.00 \\
\hline Total cholesterol & 0.0402 & 0.0122 & 0.0009 & 1.0410 & 21.6523 & 0.00 \\
\hline
\end{tabular}

$\mathrm{B}$ is the coefficient, S.E. is the Standard error, Sig. is the Significance and OR is the odds ratio.

Abbreviations: GCS- Glasgow Coma Scale; S.creatinine- Serum Creatinine; TLC- Total Leukocyte Count.

\section{DISCUSSION}

The present study was undertaken with the aim of predicting mortality in patients presenting for the first time with acute stroke with the help of routine haematological and biochemical parameters along with lipid profile.

The mean age of the patients in the study was $63.17 \pm 13.61$ years. Maximum number of cases $(n=140)$ were in the age group $\geq 60$ years. TZ Ong et al. also found mean age of 65 years in their study on acute stroke patients. ${ }^{4}$ The mean GCS of the expired patients at the time of admission was $7.52 \pm 3.89$ which was lower than discharged patients $(10.57 \pm 3.42)$. These values are statistically very significant ( $p$ value $=0.0001)$. Similar observations were made in several previous studies by TZ Ong et al. and Hamindon et al. where GCS $<9$ was an independent predictor of mortality. C. J. Weir et al. concluded that total GCS score can predict acute mortality with $88 \%$ accuracy. ${ }^{5}$ Low GCS values were associated with high mortality in studies done by Zuliani G et al. and Md. Shahriar Mahbub et al. 6,7

The mean SBP of the expired patients in the study was $164.21 \pm 25.28 \mathrm{mmHg}$ and in discharged patients $(\mathrm{n}=140)$ it was $148.30 \pm 33.41 \mathrm{mmHg}$ which was statistically significant ( $p$-value<0.05). DBP was on higher side in expired patients which was statistically significant ( $p$ value $<0.05$ ) in our study. Similar observation was found in the study conducted by $\mathrm{T} Z \mathrm{Z}$ Ong et al. where hypertension was found to be significant predictor of one month mortality. ${ }^{4}$ DževdetSmajlović et al. concluded that hypertension is an independent predictor of mortality following intracerebral haemorrhage $(\mathrm{ICH}){ }^{8}$ Sami Tetri et al. in a prospective study found that admission mean arterial blood pressure (MABP) was higher in those who died in two days ( $\mathrm{p}$-value $<0.001$ ) and within three months ( $\mathrm{p}$-value $<0.01$ ) than those who survived. High admission MABP predicted early death among the patients. ${ }^{9}$ Maria Sessa et al. studied relationship between hypertension and $\mathrm{ICH}$ in a systematic review on risk factor for ICH in general population. ${ }^{10}$ Of the 11 casecontrol and 3 cohort studies identified in the different searches for studies on hypertension as risk factor for
ICH, all showed a positive association between hypertension and ICH. Wong et al. concluded Hypertension at baseline was correlated with poor outcome at 3 months post-ICH. ${ }^{11}$ After applying multivariate logistic regression on significant clinical parameters out of all observed clinical parameters only GCS is the most important predictor of 30 day fatality in acute stroke.

Though $\mathrm{Hb}$ was found to be higher in expired patients as compared with discharged patients, it did not appear to be an independent predictor of fatality like observation of Czlonkowska. ${ }^{12}$ On the other hand Jason $\mathrm{J}$ Sico et al. observed that a very low haematocrit $(<27 \%)$ was associated with early post-stroke mortality. ${ }^{13}$ Even a moderate level of anaemia was independently associated with an increased risk of death during the first year following stroke. It is presumed that they play role by altering the cerebral blood flow. Infarct size increases due to loss vitality of penumbra by impaired oxygen supply if there is reduced $\mathrm{Hb}$ level which ultimately leads to poor prognosis. Low $\mathrm{Hb}$ level associated with larger hematoma volume which increases mortality. The mean TLC taken at the time of admission was higher in the expired patients. It was very significant when compare with discharged group ( $p$ value $=0.0001$ ). In a study by Kazmierski R et al. each 1000 cell/microliter increase in TLC at the admission was associated with increased risk for in-hospital mortality. ${ }^{14}$ Czlonkowska et al. have also demonstrated increased leukocytes as an independent predictor of 1-month case fatality in stroke patients. ${ }^{12}$ Feng-zeng $\mathrm{Li}$ et al. showed that patients with the lowest WBC count had a 30-day mortality of about $6 \%$, whereas patients with higher WBC count had a 30-day mortality of $26 \%$ to $68 \% .^{15}$ Leukocytosis may occur as a consequence of infection preceding onset of stroke or as a consequence of ischemia and brain damage. Leucocytosis influence poor prognosis through vessels plugging, release of hydrolytic enzymes, oxygen free radicals or initiation of thrombosis.

We found that PC did not show any significant difference among expired patients and discharged subgroup $(\mathrm{P}>$ 0.05). It was contrast to the study of D'Erasmo E et al. where PC obtained within 48 hours of hospital 
presentation was significantly lower in expired patient in comparison to control group. ${ }^{16}$ There was statistically significant difference in mean value of ESR in the two different subgroup ( $p$ value $=0.01$ ) which is similar to other studies. A high ESR value has been associated with large ischemic lesions and more severe deficits noted by Chamorro A et al. ${ }^{17}$ According to Rallidis LS et al, an elevated ESR value may indicate a greater increase in the concentration of fibrinogen; a more pronounced reduction in the cerebral blood flow, a larger lesion and a poorer outcome. ${ }^{18}$ ESR was found to be an independent predictor of mortality among stroke patients in a study done by Wakif et al. ${ }^{19}$ Though exact mechanism of acute increase in ESR patients suffering from ischemic stroke is unknown, but it is proposed that undiagnosed infections and inflammatory factors leads to raised ESR and high ESR is regarded as indirect sign of thrombosis. On applying Multivariate Logistic Regression on significant haematological parameters out of all observed parameters only TLC is the most important predictor of 30 day fatality in acute stroke.

During early hospitalization, regardless of a known diagnosis of diabetes, a raised RBS is known to be a significant risk factor for duration of stay in the hospital and major medical complications in patients with ischemic as well as haemorrhagic stroke. The mean RBS in the expired patients in our study was $136.60 \pm 69.49$ mg\% which was higher than discharged patients (118.79 $\pm 47.64 \mathrm{mg} \%$ ) but in contrast to other studies it was statistically not significant $(\mathrm{p}=0.39)$. Schlenk et al. showed that blood glucose levels greater than $7.8 \mathrm{mmol} / \mathrm{l}$ at the time of admission independently predicted unfavourable outcome. ${ }^{20}$ Feng-zeng li et al. also showed that hyperglycaemia was an independent predictor of mortality within 30 days. $^{15}$ Jialiu et al. found that hyperglycaemia is associated with greater hematoma expansion and poor clinical outcomes after $\mathrm{ICH}^{21}$ Diabetes predisposes to occlusive vascular disease but not to ICH. However, in haemorrhagic strokes it predisposes to larger size of hematoma and increased mortality at 30 days. In diabetics, ischemic strokes are often associated with large infarct size and poor outcome due to decreased auto regulation and changes in blood coagulability.

There were more deranged electrolytes (S.Na and S.K) in expired subgroup, but similar to observations made by Woo et al, it does not make any significant difference in survival. ${ }^{23}$ But on the other hand S.creatinine and blood urea had strong impact on fatality which was found increased in expired patients, it resembles the study of Sweileh-wm et.al and Wakif et al. ${ }^{19,22}$

In our study S.bilirubin to be higher in expired subgroup. On further analysing it was evident that this was statistically significant ( $p$ value $=0.0030$ ). In expired patients AST and ALT both were lesser as compared with discharged population of patients. But after applying unpaired $\mathrm{t}$ - test there were no significant difference in both these groups. But these observations were against the observation made by Kim et al. where AST level $>70 \mathrm{IU} / \mathrm{L}$ was identified as a predictor of stroke mortality. ${ }^{24}$ In studies there is $\mathrm{U}$ shape correlation between bilirubin and risk of stroke was shown i.e. the risk of stroke increased at both low and high S.bilirubin level. The association can be explained by a close relationship between liver dysfunction and abnormality of almost all major haemostatic parameters governing the progress of haematoma. For all biochemical parameters multivariate logistic regression applied to find out which parameter determine 30 day fatality in acute stroke then its only blood urea and S.creatinine which were found to be determinant of poor prognosis independently.

We have also taken in account lipid profile measurement in our study in which it was found that mean total cholesterol in expired patients was lower (159.80 \pm 28.69 $\mathrm{mg} / \mathrm{dL})$ than discharged patients $(234.15 \pm 55.15 \mathrm{mg} / \mathrm{dL})$ and these observations are very significant ( $p=0.0001)$. Though literature is full regarding hypercholesterolemia leading to increased incidence of atherosclerosis, and coronary and cerebral strokes, Dyker AG et al. found that low total cholesterol associated with poor outcome. ${ }^{25}$ It was also observed LDL mean value of LDL in expired patients was $71.50 \pm 27.49 \mathrm{mg} / \mathrm{dL}$ in comparison to $114.39 \pm 61.91 \mathrm{mg} / \mathrm{dL}$ in discharged patients and total cholesterol it was also statistically significant $(p=0.0200)$. S.triglyceride, HDL and VLDL values were on higher side in expired patients but none of them were statistically significant. Yih-tsen Lai et al. and Pan S-L et al. also showed that higher total cholesterol levels associated with better long-term motor function after ischemic stroke. ${ }^{27}$ When Multivariate Logistic Regression applied for lipid profile it was found that cholesterol is the only parameter from lipid profile which can predict mortality in acute stroke. Vauthey $\mathrm{C}$ et al also support our observation. ${ }^{28}$

\section{CONCLUSION}

From this study we can conclude that low GCS, higher SBP and DBP as well as increased TLC, raised ESR, increased S. bilirubin, increased blood urea, S.creatinine, low S. cholesterol and low LDL associated with poor outcome.

On the other hand $\mathrm{Hb}, \mathrm{PC}, \mathrm{RBS}, \mathrm{S} . \mathrm{Na}, \mathrm{S} . \mathrm{K}$, AST, ALT and levels of $S$.triglycerides, HDL and VLDL do not show any significant association with outcome in acute stroke patient. If these parameters taken collectively predicting outcome may become easy and it is useful to take necessary interventions accordingly in early hours focusing on correctable parameters to prevent mortality in acute stroke.

Funding: No funding sources Conflict of interest: None declared

Ethical approval: Approved by institutional ethics committee 


\section{REFERENCES}

1. Pandian JD, Srikanth V, Read SJ, Thrift AG. Poverty and stroke in India: a time to act. Stroke. 2007;38:3063-9.

2. Dalal PM, Shenoy A. Stroke Epidemic in IndiaTime to prioritize prevention strategies. JAPI. 2013.

3. Dalal P.M. Burden of stroke -Indian prospective. International J Stroke. 2006;1:164-6.

4. Ong TZ, Raymond AA. Risk Factors for Stroke and Predictors of One-Month Mortality Singapore Med J. 2002;43:517-21.

5. Weir CJ, Bradford APJ, Lees KR. The prognostic value of the components of the Glasgow Coma Scale following acute stroke. QJM. 2003; 96:67-74.

6. Zuliani G, Cherubini A, Ranzini M, Ruggiero C, Atti AR, Fellin R. Risk Factors for Short-Term Mortality in Older Subjects with Acute Ischemic Stroke Gerontology. 2006;52:231-6.

7. Miah MT, Aminul Hoque AKM, Khan RR, Nur Z, Mahbub MS, Rony RI, et al. GCS following Ac. Stroke and in hospital outcome. Journal of medicine. 2009;10:11-4.

8. Smajlović D, Salihović D, Ibrahimagić OC, Sinanović O, Vidović M. Analysis of risk factors, localization and 30-day prognosis of intracerebral hemorrhage. Bosnian journal of basic medical sciences. 2008;8:121-5.

9. Tetri S, Juvela S, Saloheimo P, Pyhtinen J, Hillbom M. Hypertension and diabetes as predictors of early death after spontaneous intracerebral haemorrhage. J Neurosurg. 2009;110:411-7.

10. MariaSessa. Intracerebral haemorrhage and hypertension. Neurol Sci. 2008;29:S258-9.

11. Wei JW, Huang Y, Wang JG, Liu M, Wong LKS, Huang Q, et al. Comparison of Recovery Patterns and Prognostic Indicators for Ischemic and Haemorrhagic Stroke in China: The China QUEST (Quality Evaluation of Stroke Care and Treatment) Registry Study. Stroke. 2010, 41:1877-83.

12. Członkowska A, Ryglewicz D, Lechowicz W. Basic analytical parameters as the predictive factors for 30-day case fatality rate in stroke. ActaNeurolScand. 1997;95:121-4.

13. Jason J Sico, Laura J Myers, Dede Ordin, Linda S Williams, Dawn M Bravata. The Association between Hematocrit and Mortality among Ischemic Stroke Patients Stroke. 2012;43:A147.

14. Kazmierski R, Guzik P, Ambrosius W, Ciesielska A, Moskal J, Kozubski W. Predictive value of white blood cell count on admission for in-hospital mortality in acute stroke patients. Clin Neurol Neurosurg. 2004;107:38-43.

15. Feng-zeng Li, Hui Chen, Yong-hong Wang, Yingjun Yang, Cheng-hua Li, Zi-he Dong. A new prognostic model and score to predict short term outcome after intracerebral haemorrhage. Scientific Research and Essays. 1992;6:2063-8.

16. D'Erasmo E, Aliberti G, Celi FS, Romagnoli E, Vecci E, Mazzuoli GF. Platelet count, mean platelet and their relation to prognosis in cerebral infarction. J Intern Med. 1990;227:11-4
17. Chamorro A, Vila N, Ascaso C, Saiz A, Montalvo J, Alonso P et al. Early prediction of stroke severity. Role of erythrocyte sedimentation rate. Stroke. 1995;26:573-6.

18. Rallidis LS, Vikelis M, Panagiotakos DB, Liakos GK, Krania E, Kremastinos DT. Usefulness of inflammatory and haemostatic markers to predict short term risk for death in ischemic stroke. ActaNeurol Scand. 2008;117:415-20.

19. El-Sheikh WM. Stroke mortality: predictive value of simple laboratory tests and APACHE III scoring system. Menoufiya Medical Journal 353 Stroke Mortality: Predictive. 2009;22.

20. Florian Schlenk, Peter Vajkoczy, Asita Sarrafzadeh. Inpatient Hyperglycaemia Following Aneurysmal Subarachnoid Haemorrhage: Relation to Cerebral Metabolism and Outcome. Neurocritical Care. 2009:56-63.

21. Jia Liu, Ben-Bo Gao, Allen C. Clermont, Price Blair, Tamie J. Chilcote, and Sukanto Sinha et al. Hyperglycaemia-induced cerebral hematoma expansion is mediated by plasma kallikrein. Nature. Medicine. 2011;17:206-10.

22. Sweileh WM. Predictors of in-hospital mortality after acute stroke: impact of renal dysfunctional int. J ClinPharmacolTher. 2008;46:637-43.

23. Woo J, Lau E, Kay R, et al. A case control study of some haematological and biochemical variables in acute stroke and their prognostic value. Neuroepidemiology. 1990;9:315-20.

24. Hyeon Chang Kim, Dae Ryong Kang, Chung Mo Nam, Nam Wook Hur, Jee Seon Shim, Sun Ha Jee et al. Elevated serum aminotransferase level as a predictor of intracerebral haemorrhage: Korea medical insurance corporation study. Stroke 2005; 36:1642-7.

25. Dyker AG, Weir CJ, Lees KR. Influence of cholesterol on survival after stroke: retrospective study. BMJ. 1997;314:1584-8.

26. Yih-tsen Lai, Ching-lin Hsieh, Hung-pin Lee, Shinliang Pan. Are higher total serum cholesterol levels associated with better long-term motor function after ischemic stroke? Nutritional Neuroscience. 2012;15:239-43.

27. Pan S-L, Lien I-N, Chen TH. Is higher serum total cholesterol level associated with better long-term functional outcomes after noncardioembolic ischemic stroke? Arch Phys Med Rehabil. 2010;91:913-8.

28. Vauthey C, deFreitas GR, van Melle G, Devuyst G, Bogousslavsky J. Better outcome after stroke with higher serum cholesterol levels. Neurology. 2000;54:1944-9.

Cite this article as: Pandey PC, Dwivedi NC, Yadav RK, Sonkar KK. Mortality in acute stroke: predictive value of lipid profile and routine investigations. Int $\mathbf{J}$ Adv Med 2015;2:235-40. 